\title{
OBSERVACIONES SOBRE LA REPRODUCCIÓN Y EL CICLO VITAL DE PLUSIOTIS COSTATA BLANCHARD, 1851 (COLEOPTERA: MELOLONTHIDAE: RUTELINAE)
}

\author{
Miguel Ángel MoRón \\ Red de Biodiversidad y Sistemática, Instituto de Ecología, A. C. Apdo. Postal 63, Xalapa, Veracruz \\ 91000, MÉXICO. E-mail: miguel.moron@inecol.edu.mx
}

Morón, M. A. 2010. Observaciones sobre la reproducción y el ciclo vital de Plusiotis costata Blanchard, 1851 (Coleoptera: Melolonthidae: Rutelinae). Acta Zool. Mex. (n. s.), 26(3): 705-720.

RESUMEN. Se describe el aparato reproductor de Plusiotis costata Blanchard. En los machos está formado por dos testículos, cada testículo con seis folículos, dos conductos deferentes, dos glándulas accesorias, el bulbo eyaculador, el conducto eyaculador y un edeago complejo. En las hembras presenta dos ovarios, cada uno con seis ovariolas, dos oviductos laterales, el oviducto común, una bursa copulatrix, la espermateca con su glándula, y la cámara genital con cuatro pares de placas genitales. También se aporta información sobre el comportamiento de cópula y el ciclo de vida bianual de $P$. costata en condiciones de cautiverio.

Palabras Clave: Anatomía, aparato reproductor, cópula, ciclo vital, rutelinos, México.

Morón, M. A. 2010. Observations on the reproduction and life cycle of Plusiotis costata Blanchard, 1851 (Coleoptera: Melolonthidae: Rutelinae). Acta Zool. Mex. (n. s.), 26(3): 705-720.

ABSTRACT. The reproductive system of Plusiotis costata Blanchard is described. The male system consist of two testes, each testis with six follicles, two vasa deferentia, two accessory glands, ejaculatory bulb, ejaculatory duct, and complex aedeagus. The female system is formed by two ovaries, each with six ovarioles and two lateral oviducts, the common oviduct, bursa copulatrix, spermatheca with its gland, and genital chamber with four pairs of genital plates. Information on copulation behavior and biannual life cycle of $P$. costata in captivity is also included.

Key Words: Anatomy, reproductive system, copulation, life cycle, shiny chafers, Mexico.

\section{INTRODUCCIÓN}

A partir de la publicación del volumen 10 de "Los Coleópteros del Mundo" (Morón 1990) se incrementó notablemente el interés por la taxonomía y la distribución de las especies de Chrysina Kirby, 1827 y Plusiotis Burmeister, 1844, al grado de que en 15 años el número de taxones descritos creció en un $75 \%$, pasando de 56 especies en 1989 a 98 especies en 2004 (Morón 1999, Hawks 2001, Monzón-Sierra 2006). Sin embargo, muy poco se ha avanzado en el conocimiento de su biología y hábitos, a pesar de la importancia que puede tener el establecimiento de criaderos con propósitos comerciales y científicos, los cuales podrían integrarse en programas para

Recibido: 19/03/2010; aceptado: 28/05/2010. 
el aprovechamiento racional de los recursos naturales en muchas localidades montañosas donde aún son abundantes estos escarabajos, y también como parte de proyectos para la protección y recuperación de especies endémicas amenazadas o en riesgo de extinción (Morón 1999, Solís 2009).

Son escarabajos eminentemente forestales, que pueden encontrarse entre los $50 \mathrm{y}$ 3,800 m de altitud. Los adultos de todas las especies conocidas pueden ser atraídos por las luces eléctricas, generalmente durante las primeras horas de la noche, y gracias a ello ha sido posible registrar su presencia en gran cantidad de localidades dentro del área comprendida entre el extremo sur de los Estados Unidos y Ecuador. Pero solo se tienen algunas observaciones esporádicas sobre sus preferencias para alimentarse y aparearse (Boucard 1875, Biederman 1907, Young 1957, Hawks 2002). Se ha generalizado que los adultos pasan la mayor parte del tiempo entre el follaje de árboles y arbustos, donde consumen el tejido foliar y forman parejas reproductoras. En algún momento, las hembras vuelan hacia el piso del bosque en busca de madera en descomposición para depositar sus huevos, como troncos derribados y tocones, donde se desarrollan las larvas hasta alcanzar la talla máxima del tercer estadio, y entonces salen de la madera para pupar en una celda endeble formada dentro del suelo subyacente; al emerger los adultos vuelan hacia las partes superiores de los árboles (Morón 1991).

Sólo se cuenta con descripciones completas de las larvas de tercer estadio de $P$. adelaida Hope, P. woodi Horn y Ch. macropus Francillon (Ritcher 1966, Morón 1976), y se han publicado diagnosis de las larvas de tercer estadio de P. lecontei Horn y Ch. erubescens Bates (Morón y Deloya 1990), todas acompañadas con observaciones breves sobre algunos aspectos de su desarrollo, pero con excepción de P. adelaida y Ch. macropus (Morón 1985) no se han obtenido datos sobre sus ciclos vitales. Ninguna pupa de estos géneros ha sido descrita. Las particularidades del comportamiento reproductor de estos rutelinos apenas se conocen, y los detalles de la anatomía en larvas y adultos no han sido estudiados, con excepción de las partes esclerosadas de los genitales masculinos y femeninos, que se emplean como caracteres taxonómicos valiosos para la identificación específica (Morón 1990).

Los objetivos del presente artículo consisten en describir el aparato reproductor en los dos sexos de Plusiotis costata Blanchard, así como aportar información sobre su comportamiento copulatorio y ciclo de vida en condiciones de cautiverio.

\section{MATERIAL Y MÉTODOS}

Durante 1989 y 1990 se capturaron un total de 34 ejemplares de $P$. costata atraídos por las luces del alumbrado público de los jardines del Instituto de Ecología, A. C., situado $2.5 \mathrm{~km}$ al S de Xalapa, Veracruz, México, a 1,340 m de altitud (19³0'46.54" $\left.\mathrm{N}, 96^{\circ} 56^{\prime} 36.26^{\prime} \mathrm{O}\right)$. En julio de 1989, seis machos y cuatro hembras fueron confinados en un terrario de vidrio (50 cm longitud, $30 \mathrm{~cm}$ anchura y $40 \mathrm{~cm}$ de altura) 
provisto con una capa de tierra forestal de $12 \mathrm{~cm}$ de espesor, un trozo grande de madera podrida de Liquidambar styracyflua L. (Hamamelidaceae), y un frasco de vidrio con agua para sostener ramas frescas de Carpinus caroliniana Walter (Betulaceae), Quercus xalapensis Humboldt \& Bonpland (Fagaceae) y L. styraciflua. Los terrarios se revisaron tres veces al día (12:00, 18:00, 20:00) durante diez semanas para observar el comportamiento de los escarabajos, tomar notas, realizar bocetos y captar fotografías con un equipo Praktica MTL3 reflex.

Cuando se confirmó la presencia de los primeros huevos en los terrarios, se colocó cada uno en caja de Petri desechable de $5.5 \mathrm{~cm}$ de diámetro provista con una mezcla a partes iguales de tierra forestal húmeda finamente cernida y aserrín de liquidámbar, y allí se observó su desarrollo hasta que alcanzaron el crecimiento medio del segundo estadio, entonces se les cambió a cajas de Petri desechables de 9.5 $\mathrm{cm}$ de diámetro con trozos pequeños de madera podrida húmeda de liquidámbar, para que continuaran su desarrollo hasta adulto. Durante este proceso se fijaron muestras de cada estadio en solución de Pampel. Otros huevos se dejaron dentro del suelo de los terrarios, donde siguieron su desarrollo. En agosto de 1990 se repitió la operación con 12 machos y seis hembras repartidos en dos terrarios.

En julio de 1989 se apartaron tres parejas recién capturadas para efectuar disecciones en fresco y dibujar sus órganos reproductores in situ antes de extraerlos para extenderlos en una caja de Petri con agua destilada y dibujarlos en ese momento. Para estas actividades se empleó un estereomicroscopio Carl Zeiss SV6 con 12 a 75 aumentos, provisto con cámara lucida, reglilla ocular y lámpara de luz fría. Se siguió la terminología anatómica de Sharp \& Muir (1912), Snodgrass (1935), Böving (1942), Matsuda (1976), D'Hotman \& Scholtz (1990) y Carrillo-Ruiz et al. (2008). Se depositaron muestras de los adultos y los estados inmaduros estudiados en la Colección Entomológica del Instituto de Ecología, A. C. (IEXA) en Xalapa, Veracruz.

\section{RESULTADOS}

Los adultos de $P$. costata son de color verde brillante con reflejos dorados o amarillos en los bordes de los apéndices; miden entre 28 y $31 \mathrm{~mm}$ de longitud (borde del clípeo a la placa pigidial), 16 a $19 \mathrm{~mm}$ de anchura humeral y 10 a $11 \mathrm{~mm}$ entre el mesosternón y la superficie dorsal de los élitros. En los dos sexos la mayor parte de la región ventral de la cavidad abdominal está ocupada por los órganos reproductores doblados en forma intrincada, en tanto que por la región dorsal se acomodan las asas del mesenterón y el proctodeo, que en conjunto alcanzan una longitud de 130-145 $\mathrm{mm}$, con diámetro de 0.7 a $3.8 \mathrm{~mm}$. El intestino cuenta con cuatro túbulos de Malpighi bipectinados insertos en la región pilórica, que recorren toda la longitud del mesenterón y el proctodeo. En la mitad anterior del mesenterón estos túbulos se encuentran entretejidos con numerosas traqueolas y pequeños sacos aéreos. 


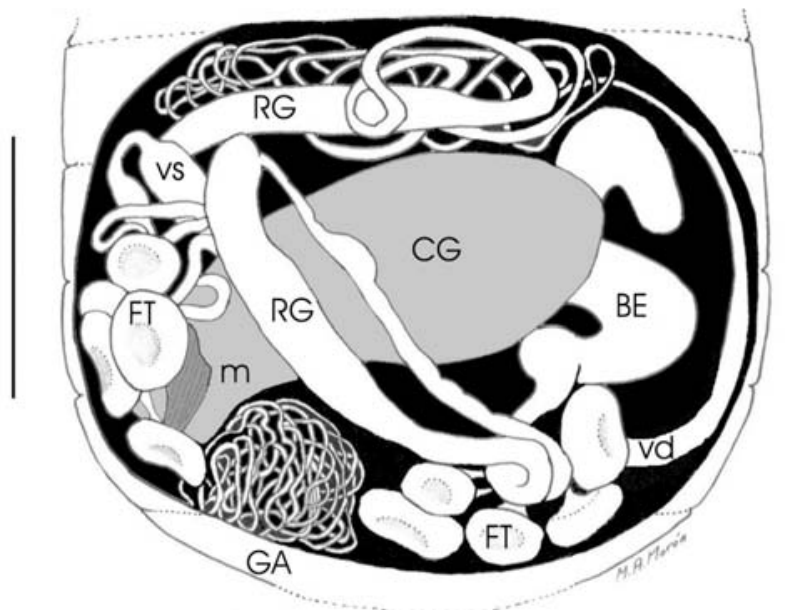

Figura 1. Aspecto ventral de la cavidad visceral de un macho de Plusiotis costata, con todos los órganos en posición natural. BE bulbo eyaculador, CG cápsula genital, FT folículo testicular, GA glándula accesoria, m músculo, RG reservorio glandular, Vd vaso deferente, Vs vesícula seminal. Escala $5 \mathrm{~mm}$.

Anatomía del aparato reproductor masculino. En disección desde la parte ventral del abdomen, in situ destaca el gran tamaño de la cápsula genital (longitud $9.8 \mathrm{~mm}$ ), que ocupa un $70 \%$ de la anchura de la cavidad abdominal $(13 \mathrm{~mm})$. En reposo dicha estructura está dispuesta en sentido transversal, con su lado izquierdo hacia el extremo distal del abdomen y los parámeros hacia el lado izquierdo del abdomen (Fig. 1), por lo cual para prepararse a la cópula es necesario que su eje longitudinal efectúe una rotación de $90^{\circ}$ junto con un movimiento de retracción de la falobase hacia la parte media del interior del último esternito, frente a la abertura ano-genital.

El aparato consta de un par de testículos con dos vasos deferentes y vesículas seminales, un par de glándulas accesorias con reservorios y conductos, un bulbo y un ducto eyaculatorio que termina en el edeago o saco interno, contenido en una gran cápsula esclerosada (Fig. 2). Los testículos están formados por seis folículos redondeados y deprimidos, que en promedio miden $3.2 \mathrm{~mm}$ de diámetro cada uno; los seis vasos eferentes son muy angostos, un poco más largos que cada folículo y se insertan en su respectivo vaso deferente, el cuál incrementa notablemente su calibre $(0.6 \mathrm{~mm})$ en la parte media de su longitud, y forma tres asas antes de comunicarse con una vesícula seminal conformada por dos cámaras globulares de distinto tamaño separadas por un pasaje corto. La parte basal de ésta vesícula es estrecha hasta el punto de unión con el reservorio de la glándula accesoria respectiva. Cada glándula alcanza hasta $116 \mathrm{~mm}$ de longitud, con un diámetro promedio de $0.2 \mathrm{~mm}$, y en su mayor parte se encuentra enrollada sobre sí misma. El bulbo eyaculador es 


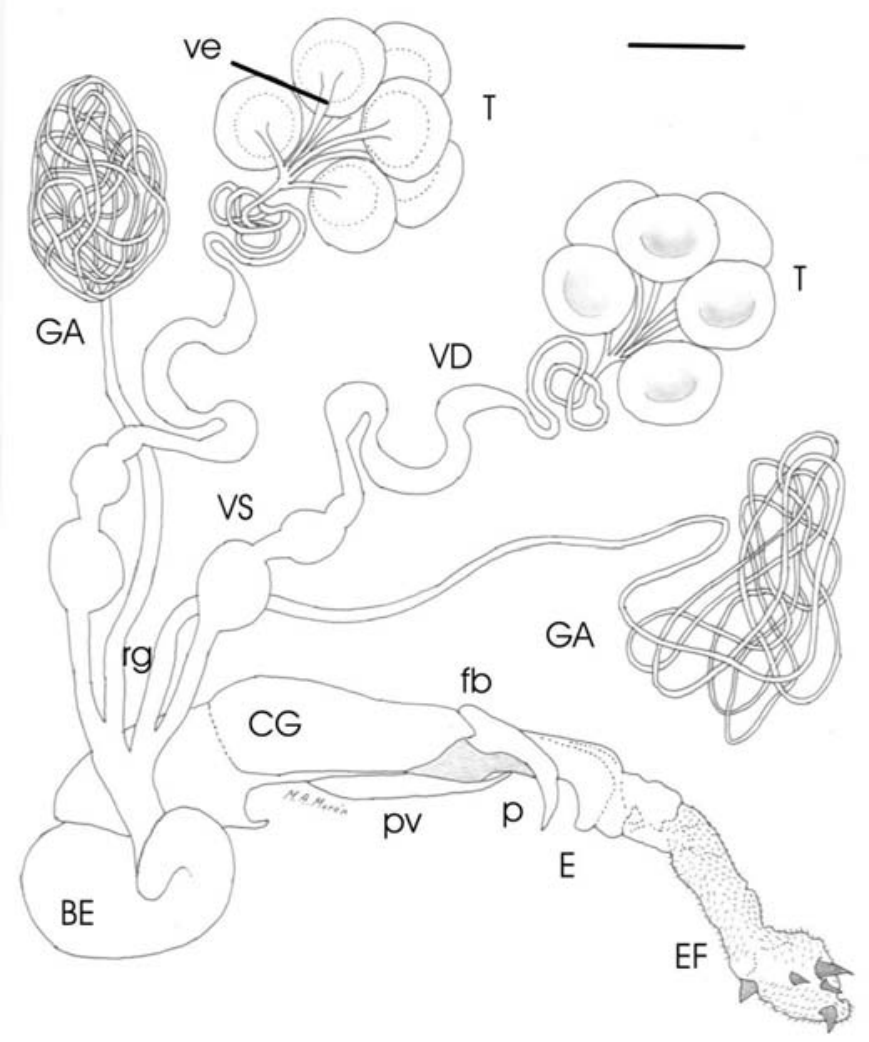

Figura 2. Aparato reproductor masculino de Plusiotis costata extendido. BE bulbo eyaculador, CG cápsula genital, E edeago, EF endofalo, fb falobase, GA glándula accesoria, p parámero, pv placa ventral, rg reservorio glandular, T testículo, ve vaso eferente, VD vaso deferente, VS vesícula seminal. Escala $5 \mathrm{~mm}$.

voluminoso, asimétrico, notablemente recurvado después de la inserción con la vesícula seminal y el reservorio glandular (Fig. 2).

El edeago o saco interno es muy largo y se aloja invaginado dentro de la cápsula esclerosada. La mitad distal del saco membranoso (endofalo) extendido tiene 8 o 9 dentículos esclerosados preapicales (cornuti) dispuestos de modo irregular, cada dentículo tiene forma diferente, miden entre $0.52-0.78 \mathrm{~mm}$ de longitud y $0.32-0.46 \mathrm{~mm}$ de anchura máxima (Fig. 3). Toda la membrana del ápice y parte de la membrana ventral de la región media del saco extendido (endofalo) está cubierta con microespinas, y por la región dorsal presenta gránulos esclerosados. La membrana de la porción basal del saco no tiene microestructuras, pero en la región dorsal tiene un par de laminillas esclerosadas alargadas internas que pueden corresponder con las temonas. 


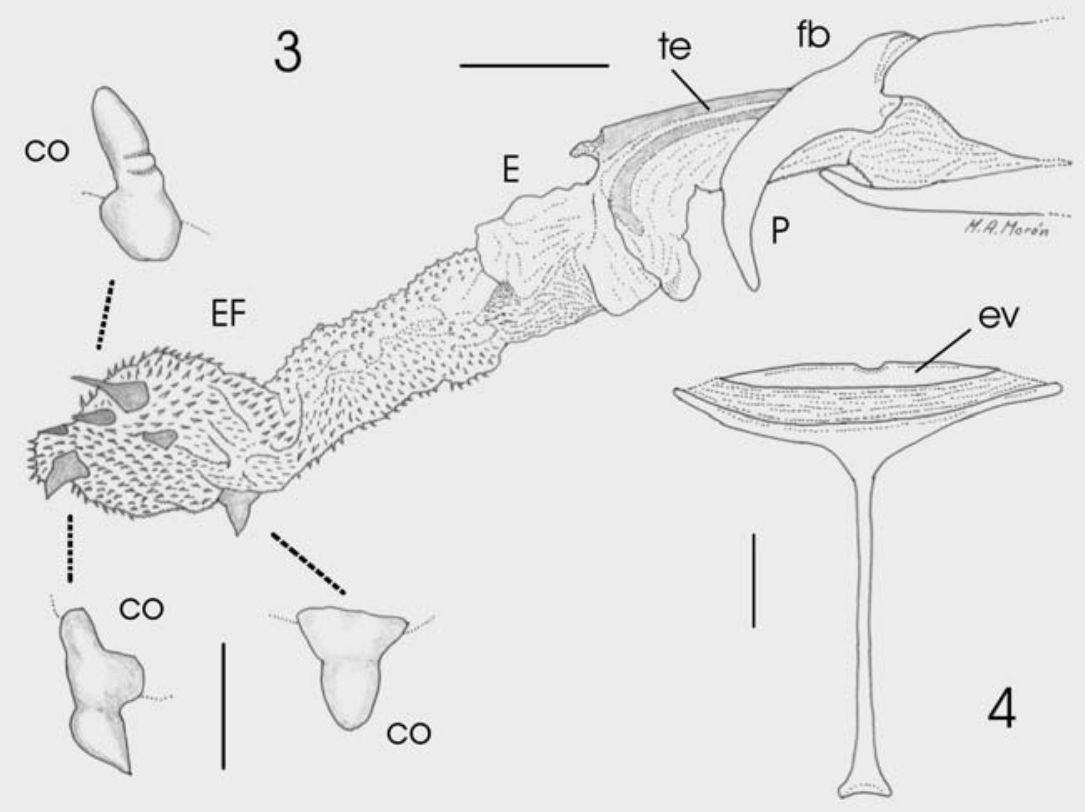

Figuras 3-4. Estructuras internas de un macho de Plusiotis costata. 3. Detalles del aparato copulador. Escala $0.5 \mathrm{~mm}$. 4. Aspecto dorsal del spiculum gastrale. Escala $1 \mathrm{~mm}$. co cornuti, E edeago, EF endofalo, ev esclerito vestigial, fb falobase, P parámero, te temonas.

La cápsula genital es del tipo bilobulado (sensu D’Hotman \& Scholtz, 1990) muy esclerosada, de color pardo amarillento con reflejos iridiscentes rojizos sobre todo en los parámeros, y mide $9.8 \mathrm{~mm}$ de largo por $4.4 \mathrm{~mm}$ de ancho máximo. Consta de una pieza basal corta y redondeada, tectum semicilíndrico, ligeramente estrecho hacia su extremo distal, con dos prominencias dorso-basales; la falobase está ampliamente articulada con el tectum por medio de una membrana poco esclerosada y da soporte a un par de parámeros simétricos curvados dorso-ventralmente, un poco divergentes, con dentículos preapicales en sus bordes internos (Fig. 6). Por su región ventral la cápsula tiene una placa esclerosada amplia, convexa, provista de grandes espinas laterales que sobresalen en los ángulos distales, flanqueando un borde membranoso. En vista ventral son aparentes los ligamentos tractores de la falobase que facilitan el movimiento de los parámeros (Fig. 5). Una vista lateral de la cápsula permite apreciar la curvatura de los parámeros y de las espinas apicales de la placa ventral, así como los cóndilos ventro-laterales de la pieza basal, donde se apoyan los ligamentos para mover toda la cápsula. Algunos movimientos del órgano copulador se realizan con apoyo en los músculos y membranas asociadas con el spiculum gastrale, que tiene una 
base larga muy delgada y brazos laterales medianos estrechos, dispuesto en forma de "T" en cuyo borde dorsal está articulado un esclerito vestigial (Fig. 4).

Anatomía del aparato reproductor femenino. En disección desde la parte ventral del abdomen, in situ destacan la bursa copulatrix (longitud $10.5 \mathrm{~mm}$, ancho máximo $7.8 \mathrm{~mm}$ ) y la corona de músculos que rodea la cámara genital. A la fecha de la disección aún no se iniciaba el desarrollo de los ovocitos, aunque la turgencia de la bursa copulatrix indicaba que ya se había efectuado al menos una cópula.

El aparato consta de un par de ovarios con dos oviductos laterales, un oviducto común, una bursa copulatrix, una espermateca con su glándula, una cámara genital provista de
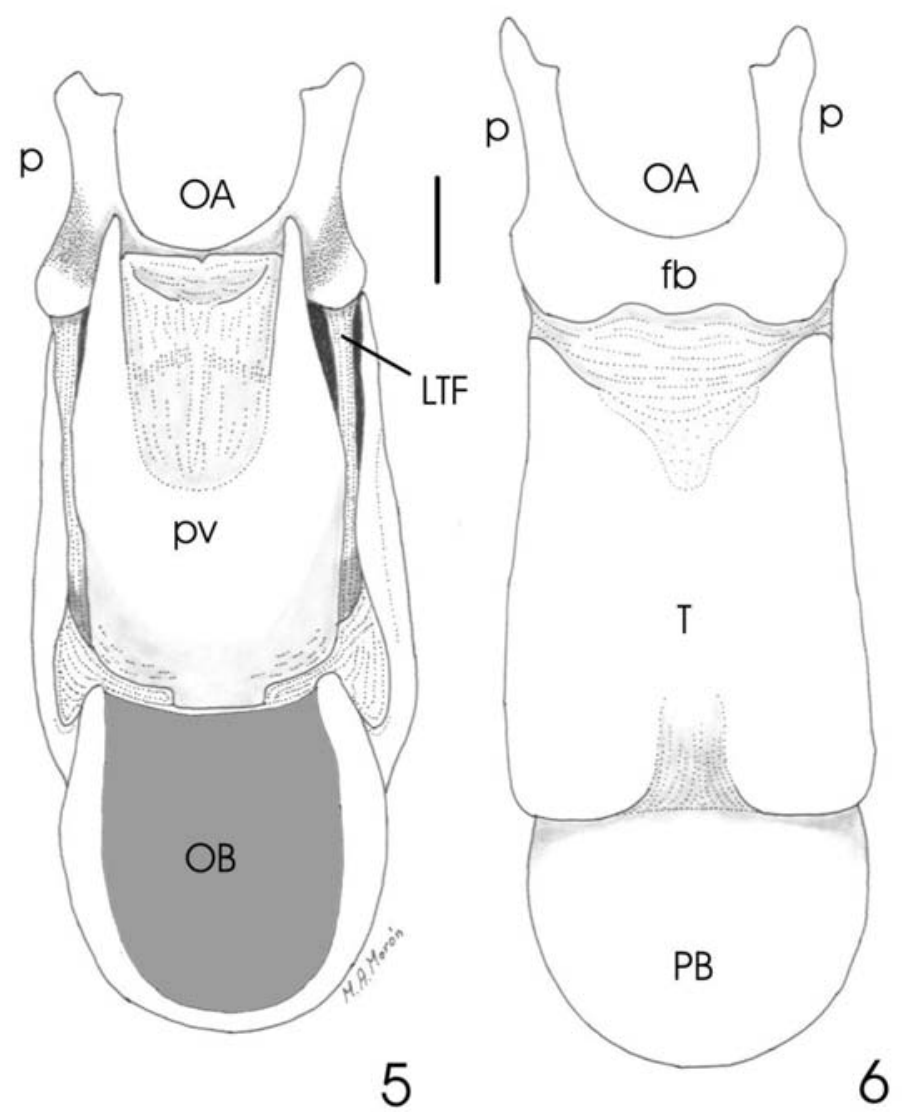

Figuras 5-6. Cápsula genital masculina de Plusiotis costata. 5. Aspecto ventral. 6. Aspecto dorsal. Escala $1 \mathrm{~mm}$. LTF ligamento tractor de la falobase, fb falobase, OA ostium apical, OB ostium basal, $\mathrm{P}$ parámeros, PB pieza basal, pv placa ventral, T tectum. 
placas esclerosadas (Fig. 7). Los ovarios están formados por seis ovariolas libres, sin membrana envolvente, que en promedio miden $11.8 \mathrm{~mm}$ de longitud cada una; los seis filamentos terminales se fusionan en un filamento apical que les mantiene reunidas. El germario y el vitelario de cada ovariola se aprecian poco diferenciados, y cada vitelario tenía evidencias de contener tres o cuatro oocitos de tamaño similar aún no desarrollados. Los pedicelos de cada ovariola son muy cortos y desembocan en un oviducto lateral ancho con dos constricciones. El oviducto común es ancho, con una longitud de 6.0-6.5 mm, y cerca de su extremo basal al lado derecho se inserta el conducto de la espermateca, estrecho, largo, sinuoso que se ensancha abruptamente en una espermateca casi globular (Fig. 7). La glándula de la espermateca no es voluminosa y sólo un poco más larga que la

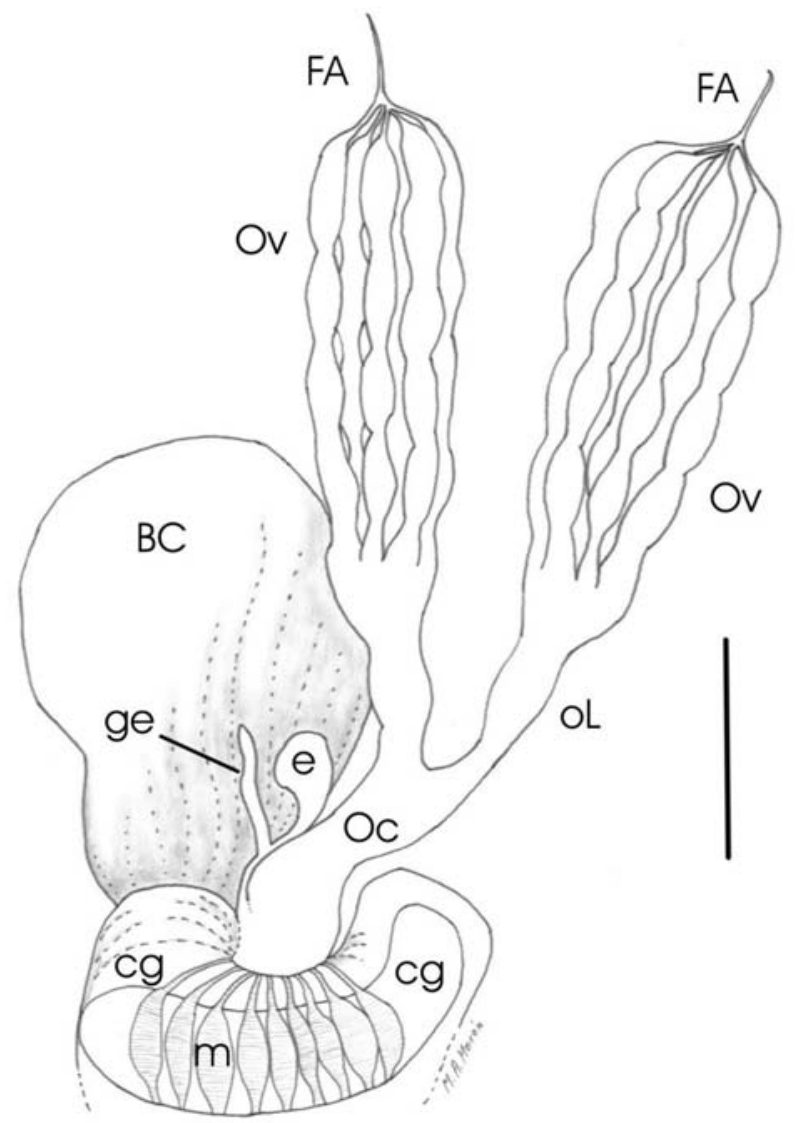

Figura 7. Aparato reproductor femenino de Plusiotis costata extendido. BC bursa copulatrix, cg cámara genital, e espermateca, FA filamento apical, ge glándula de la espermateca, m músculos, OC oviducto común, OL oviducto lateral, OV ovariola. Escala $5 \mathrm{~mm}$. 
misma espermateca. La bursa copulatrix es grande y amplia, con recubrimiento esclerosado evidente en su parte mesial. El oviducto común, el conducto de la espermateca y la bursa copulatrix desembocan casi simultáneamente en una cámara genital amplia, más ancha que larga, revestida por láminas musculares delgadas, en cuyo interior se ubican las placas genitales muy esclerosadas características de esta especie (Fig. 8).

La hembra de $P$. costata tiene cuatro pares de placas genitales, posiblemente derivadas de terguitos y esternitos vestigiales, muy esclerosadas y articuladas por membranas que les permiten cierto grado de movimiento impulsado por los músculos que rodean la cámara genital. Al tirar de las partes internas de la cámara, a través de orificio genital, se logran evaginar parcialmente estas placas, a las más expuestas les llamamos placas ventrales externas y posiblemente corresponden a modificaciones del penúltimo esternito, alcanzan $2 \mathrm{~mm}$ de anchura, $4 \mathrm{~mm}$ de longitud y muestran proyecciones laminares estrechas, divergentes, provistas con sedas finas (Fig. 8). Debajo de ellas se ubica otro par de placas mucho más pequeñas, con un extremo aguzado divergente y sedas finas a lo largo de sus bordes, a las que denominamos como placas ventrales internas, que pueden representar derivados del último esternito. A continuación se encuentra el acceso hacia la bursa copulatrix, y un par de placas esclerosadas gruesas, de $3 \mathrm{~mm}$ de anchura, con concavidades amplias, a las que llamamos placas dorsales, cada una de ellas articulada lateralmente con otra placa más pequeña y sencilla, que en conjunto pueden provenir del penúltimo y el último terguitos muy modificados (Fig. 8).

Comportamiento copulatorio. En cautiverio, tanto machos como hembras, permanecieron quietos la mayor parte del día, asegurados a las partes inferiores de las hojas y ramas delgadas. Hacia las 18:30 h (horario normal del centro de México durante julio 1989) iniciaron el consumo de las hojas de Carpinus o Quercus, en forma casi continua hasta el amanecer (06:00-06:30). Las hojas de Liquidambar no fueron consumidas. Sin mediar ningún estímulo aparente, los machos se acercaron a las hembras durante el día o la noche y trataron de montarlas, y fueron rechazados la mayoría de las veces. Cuando las hembras aceptaron el acercamiento se observaron dos variantes en la posición de acoplamiento:

a) Conforme el macho se sube al dorso de la hembra apoyando sus tarsos sobre los bordes laterales de los élitros y las mesotibias, la hembra se detiene de una rama con los tres pares de tarsos. Cuando el macho logra una postura adecuada para exponer su órgano genital frente al orificio femenino, la hembra deja de apoyar los metatarsos en la rama y deja extendidas las patas posteriores brevemente, hasta que el macho sujeta las metatibias y los metatarsos de la hembra con sus mesotarsos; y metatarsos respectivamente, al tiempo que introduce el ápice de los parámeros en la cámara genital femenina (Fig. 9). En este caso ambos individuos realizan la cópula con sus regiones ventrales hacia arriba. 


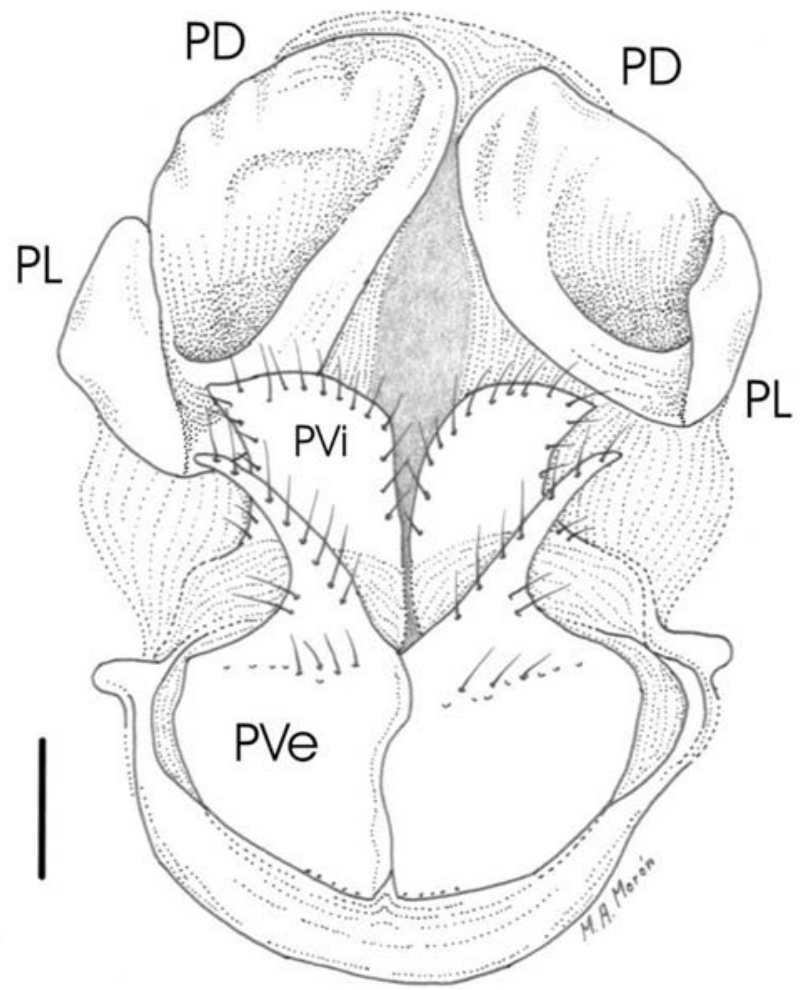

Figura 8. Placas genitales femeninas de Plusiotis costata. PD placa dorsal, PL placa lateral, PVe placa ventral externa, PVi placa ventral interna. Escala $1 \mathrm{~mm}$.

b) Similar al anterior, pero el macho se sitúa en el dorso de la hembra apoyando sus tarsos sobre los bordes laterales de los élitros, las mesotibias y las metatibias de su pareja, la hembra se sostiene de una rama por medio de los tres pares de tarsos. Ya que el macho alcanza la postura adecuada para exponer su edeago, progresivamente la hembra deja de apoyar los meso y metatarsos en la rama hasta quedar suspendida sólo con los protarsos, en tanto que el macho sujeta las metatibias y metatarsos de la hembra con sus mesotarsos y metatarsos respectivamente, en el momento que inicia la introducción del ápice de los parámeros. En esta situación la cópula se desarrolla con las cabezas de los dos individuos dirigidas hacia arriba.

Independientemente de cómo se sujete la hembra a la planta, para dar inicio a la cópula el macho apoya siempre sus protarsos sobre la mitad anterior del borde epipleural de cada élitro femenino, sus mesotarsos aseguran la mitad distal de las metatibias de la hembra, y los metatarsos masculinos se enganchan con los metatarsos femeninos. A pesar del notable volumen de la cápsula genital masculina, se introducen por completo 


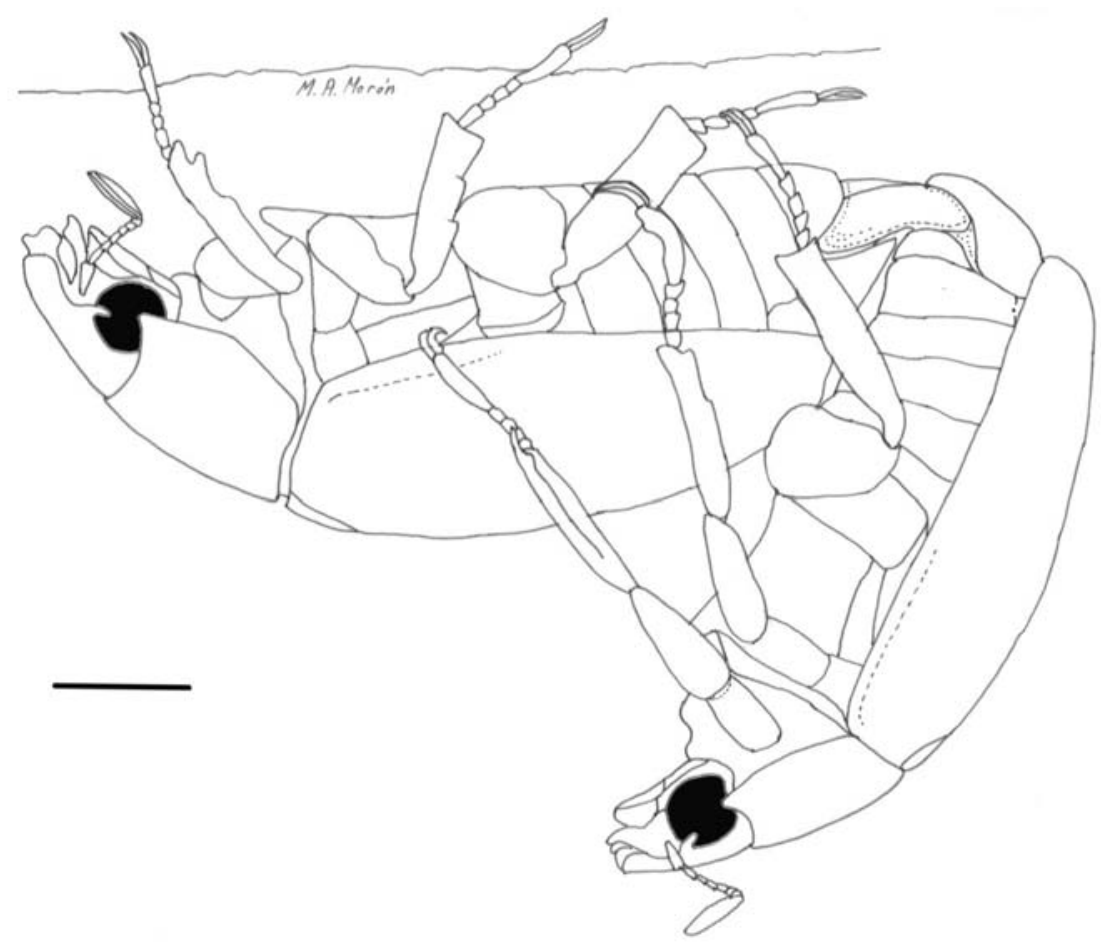

Figura 9. Pareja de Plusiotis costata mostrando la postura característica de cópula bajo una rama de Carpinus caroliniana. Escala $5 \mathrm{~mm}$.

los parámeros y la falobase en la cámara genital femenina. En el interior de dicha cámara (Fig. 10) se sobreponen las proyecciones de las placas ventrales internas de la hembra con las mitades distales de los parámeros, mientras que las espinas laterales de la placa esclerosada ventral del macho se apoyan en las concavidades de las placas dorsales de la hembra, mientras que el endofalo se extiende hasta alcanzar el interior de la bolsa copulatriz, donde se descarga una mezcla blanquecina espesa formada por espermatozoides y el producto de las glándulas accesorias.

No se observaron movimientos de cortejo o estimulación previos o simultáneos con el acoplamiento, pero todo el proceso requiere bastante tiempo. El 28 de agosto de 1989 se observó que la cópula de dos parejas tuvo una duración de 4 horas (12:0016:00h), y el 16 de julio de 1990 otras dos parejas permanecieron unidas durante 2:30 horas (19:30-22:00h). Además de la posible lentitud en la transferencia espermática, una parte del tiempo de acoplamiento podría corresponder a un plazo de guardia postcopulatorio. 


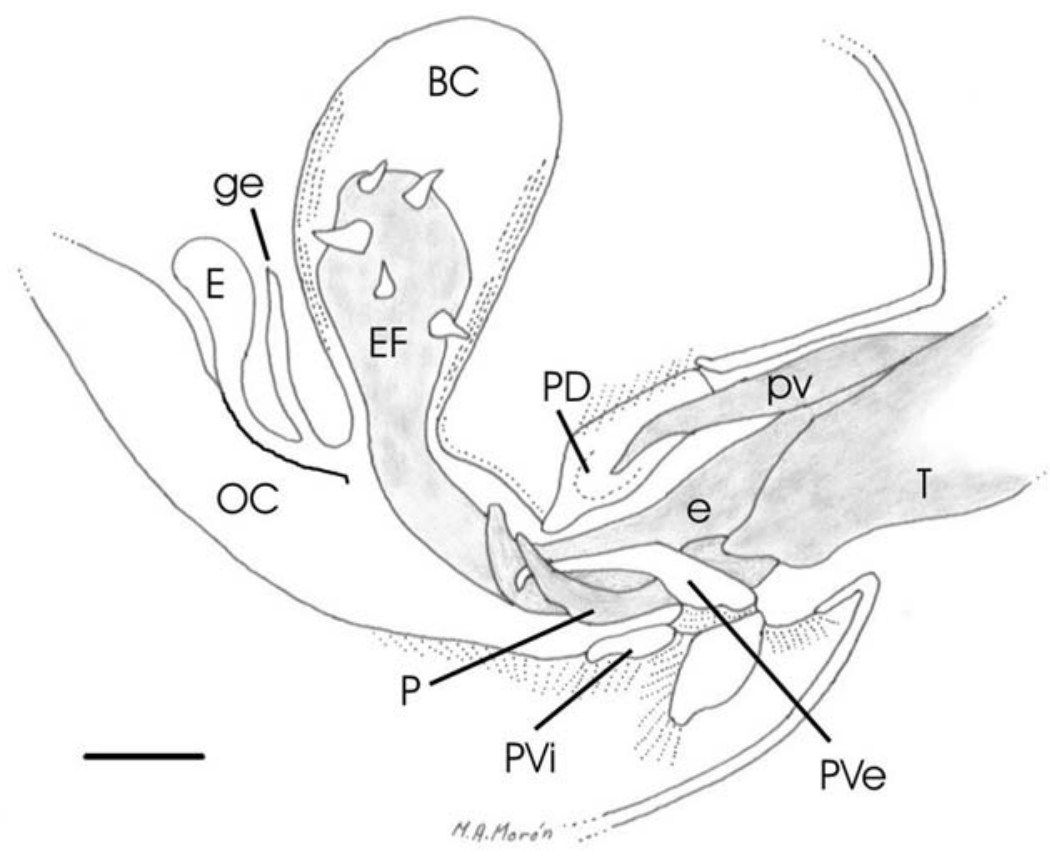

Figura 10. Interpretación gráfica del acoplamiento interno en una pareja de Plusiotis costata. BC bursa copulatrix, e edeago, E espermateca, EF endofalo, ge glándula de la espermateca, OC oviducto común, $\mathrm{P}$ parámero, PD placa dorsal femenina, pv placa ventral masculina, PVe placa ventral externa, PVi placa ventral interna, $T$ tectum. Escala $2 \mathrm{~mm}$.

Ciclo de vida. El 13 de septiembre de 1989 se encontraron 10 huevos en distintas etapas de maduración esparcidos entre el suelo del terrario (profundidad 5-10 cm), así como dos larvas de primer estadio (una recién emergida con la cabeza aún no esclerosada, y otra más desarrollada), los cadáveres de tres hembras y cinco machos, y una hembra viva. Para el 2 de octubre siguiente 8 de los 10 huevos eclosionaron y dos tenían aspecto de continuar el desarrollo embrionario. Las larvas de primer estadio se alimentaron durante 90-100 días para mudar al segundo estadio durante enero de 1990. Durante el mes de marzo siguiente algunas larvas alcanzaron el tercer estadio inicial, pero su desarrollo continuó durante 13 meses hasta que alcanzaron su talla máxima antes de iniciar el período de pupación en mayo de 1991. El 8 de julio de 1991 se obtuvieron dos hembras adultas, con lo cuál se completó un ciclo de dos años. Las larvas crecen bastante pero lo hacen lentamente, ya que en un lapso de 10 meses pasaron de 0.068-0.092g de peso fresco en vivo (larva 1) a 2.57-3.96g (larva 3 ), con un incremento de peso promedio de $3.18 \mathrm{~g}$, equivalente a 40 veces su peso inicial. 


\section{DISCUSIÓN}

La anatomía del aparato reproductor masculino del rutelino Plusiotis costata es parecida a la de los hoplinos Hoplia subcostata Bates y H. squamifera Burmeister, estudiada por Carrillo-Ruiz et al. (2008) pero tiene grandes diferencias en la complejidad de la cápsula genital (edeago, saco interno, endofalo) y en la estructura del spiculum gastrale. También es semejante al del melolontino Phyllophaga anxia (Leconte) descrito por Berbert y Helms (1972) y al del dinastino Megasoma elephas (Fabricius) (Morón, datos inéditos) con diferencias en la cápsula genital y en la forma del spiculum.

Con respecto a otros rutelinos, Micó (2001) describió la morfología de la genitalia ectodérmica en machos y hembras de varias especies europeas de anomalinos como Anomala devota (Rossi), Blitopertha lineata (Fabricius), Exomala campestris (Latreille), Mimela rugatipennis (Gräells), Phyllopertha horticola (Linné) y Anisoplia baetica Erichson, pero no proporcionó datos sobre la parte endodérmica, por lo cuál al comparar tales estructuras con las de Plusiotis costata sólo podemos decir que las diferencias en la anatomía de los aparatos masculinos se concentran en la cápsula genital, y en particular de los sacos internos, que también muestran accesorios esclerosados y divertículos con formas específicas.

La anatomía del aparato reproductor femenino de $P$. costata es parecida a la de las especies de Hoplia citadas, pero difiere en las proporciones del oviducto común, la glándula de la espermateca, la bursa copulatrix y, sobre todo, en la complejidad de las placas esclerosadas de la cámara genital. También se asemeja al de Phyllophaga anxia (Berbert y Helms 1972) y al de Megasoma elephas (Morón, datos inéditos) con diferencias en el tamaño de la glándula de la espermateca, las proporciones y el grado de esclerosamiento de las paredes internas de la bursa copulatrix, así como en el número y la configuración de las placas genitales. En comparación con los anomalinos europeos estudiados por Micó (2001) P. costata muestra diferencias en las proporciones de la espermateca y su glándula, el tamaño y esclerosamiento de la bursa, y sobre todo en la complejidad de las placas genitales.

En síntesis, aunque la anatomía de la parte endodérmica del aparato reproductor masculino y femenino de $P$. costata no muestra diferencias importantes con respecto a otras de las escasas especies de Melolonthidae cuya anatomía se haya descrito, la morfología de los órganos copuladores sí ofrece numerosas características de una mayor complejidad, principalmente derivadas de la hipertrofia y grado de esclerosamiento de estructuras que son más simples en otros grupos de lamelicornios. La enorme cápsula genital masculina es un órgano desproporcionado para la talla corporal de $P$. costata, ya que es equivalente al $35 \%$ de la longitud total del cuerpo, mientras que en Hoplia subcostata es de 16\%, en Megasoma elephas de 17\%, y en Phyllophaga anxia de $24 \%$. Además, la parte distal de dicha cápsula está provista con estructuras esclerosadas afiladas o falciformes muy desarrolladas, que tienen bastante 
correspondencia con sus contrapartes funcionales, las placas esclerosadas ubicadas en el interior de la cámara genital femenina (Figs. 3, 5-6, 8, 10).

Es posible que este complejo mecanismo de engarce sexual cumpla con otras tres funciones además de la mera transferencia espermática: 1) sujetar a la pareja firmemente en un ambiente inestable, como lo son las ramas delgadas y las hojas en la copa de un árbol, durante el tiempo necesario para culminar la transferencia de esperma; 2) dificultar la separación de la pareja por parte de otros machos competidores, factor importante en poblaciones donde predominan los machos, como ocurre en $P$. costata; y 3 ) actuar como una barrera selectiva de naturaleza morfológica ("llave-cerradura") que limite el cruzamiento entre especies afines en poblaciones simpátricas o parapátricas, barrera importante en especies sin un mecanismo de comunicación química sexual intraespecífico, o sin patrones de comportamiento precopulatorio selectivos (sensu Eberhard 1993a,b).

Hasta donde se sabe, la mayoría de las especies de los géneros Plusiotis, Hoplia, Megasoma y Phyllophaga, realizan su acoplamiento sexual en las ramas de los árboles y arbustos o sobre tallos de herbáceas, por lo que podrían requerir de la primera función arriba descrita, aunque muchas de ellas no presentan un aparato copulador tan grande o complejo. En varias especies de Plusiotis y Phyllophaga se ha observado que los machos competidores empujan con la cabeza y pronoto a los machos que están iniciando una cópula en las ramas, y tratan de separar a la pareja con éxito variable (Morón obs. pers.), con lo cuál podría confirmarse la segunda función. No se ha publicado ningún registro sobre feromonas o atrayentes sexuales en especies de Plusiotis o Chrysina, y tampoco se ha registrado algún comportamiento precopulatorio, por lo cuál una barrera morfológica genital podría ser una opción para aislar especies vecinas. En cautiverio se observó que un macho de Plusiotis adelaida Hope intentó copular con una hembra de P. costata, pero aunque completó el proceso de montura e introdujo el ápice del edeago, no logró continuar el acoplamiento, y en pocos minutos desistió (Morón obs. pers.). En condiciones naturales estas especies habitan ambientes diferentes, pero con frecuencia colindantes o sobrelapados en el bosque mesófilo de montaña, los encinares y los pinares, pero las características de sus aparatos copuladores son muy diferentes (Morón 1990), casi están en los extremos opuestos de la diversidad genital observada en el género; sin embargo, algo atrajo al macho en cuestión hacia la hembra de la otra especie, pero al parecer las grandes diferencias de sus órganos copuladores impidieron una transferencia espermática que pudo haber producido una descendencia híbrida.

Con respecto a la larga duración del acoplamiento observada en $P$. costata puede compararse con lo que ocurre en especies de Macrodactylus (Melolonthinae) como M. mexicanus Burmeister, donde según Carrillo y Gibson (1960) los machos permanecen sobre las hembras "algunas horas o de uno a dos días" pero, de acuerdo 
con las observaciones de Eberhard (1993b) con M. costulatus tal vez la mayor parte de ese tiempo corresponda a una guardia precopulatoria con cortejo y otra guardia postcopulatoria, para evitar ceder la hembra a otro macho o evitar la posibilidad de una competencia espermática, considerando que los machos son abundantes y que los individuos alcanzan grandes concentraciones tal vez por efecto de algún compuesto volátil de origen vegetal propiciado por su actividad de alimentación. Un comportamiento parecido se ha documentado en el cetonino Neoscelis dohrni Westwood (Nogueira et al. 2004), cuyos machos defienden territorios de alimentación y custodian hembras antes de iniciar la cópula.

Es necesario estudiar en forma comparativa la anatomía y el comportamiento reproductor de más especies de Melolonthidae representativas de las distintas subfamilias y tribus, para definir otros caracteres útiles en los análisis sobre la filogenia de los Scarabaeoidea y la evolución del comportamiento reproductivo.

AGRADECIMIENTOS. A César V. Rojas por su colaboración técnica en distintos aspectos de este trabajo, que representa una contribución a la línea de investigación "Sistemática y ecología de los Coleópteros Lamelicornios de América Latina" apoyada con recursos del Instituto de Ecología, A.C. (cuenta 20030-10011).

\section{LITERATURA CITADA}

Berberet, R. C. \& T. J. Helms. 1972. Comparative anatomy and histology of selected systems in larval and adult Phyllophaga anxia (Coleoptera: Scarabaeidae). Annals Entomological Society of America, 65(5): 1026-1053.

Biederman, C. R. 1907. Notes on Plusiotis beyeri Skinner. Entomological News, 1907: 7-9.

Boucard, A. 1875. Monographic list of the Coleoptera of the genus Plusiotis of America, north of Panama, with description of several new species. Proceedings of the Zoological Society of London, 1875: 117-125.

Böving, A. G. 1942. A classification of larvae and adults of the genus Phyllophaga (Coleoptera: Scarabaeidae). Memoirs of the Entomological Society of Washington, 21: 1-96.

Carrillo, J. L. \& W. W. Gibson. 1960. Repaso de las especies mexicanas del género Macrodactylus (Coleoptera: Scarabaeidae) con observaciones biológicas de algunas especies. Oficina de Estudios Especiales, SAG, México. Folleto Técnico 39. 102 pp.

Carrillo-Ruiz, H., I. Martínez \& M. A. Morón. 2008. Comparative study of the reproductive system of two species of Hoplia (Coleoptera: Scarabaeidae: Hopliinae). Proceedings of the Entomological Society of Washington, 110(3): 778-788.

D'Hotman, D. \& C. H. Scholtz. 1990. Phylogenetic significance of the structure of the external male genitalia in the Scarabaeoidea (Coleoptera). Entomology Memoir Department of Agricultural Development Republic of South Africa, No. 77: 1-51.

Eberhard, W. G. 1993a. Copulatory courtship and morphology of genitalic coupling in seven Phyllophaga species (Coleoptera: Melolonthidae). Journal of Natural History, 27: 683-717.

Eberhard, W. G. 1993b. Copulatory courtship and genitalic mechanics of three species of Macrodactylus (Coleoptera: Scarabaeidae Melolonthinae). Ethology, Ecology \& Evolution, 5: 19-63.

Hawks, D. C. 2001. Taxonomic and nomenclatural changes in Chrysina and a synonymic checklist of species (Scarabaeidae: Rutelinae). Occasional Papers of the Consortium Coleopterorum, 4: 1-8. 
Hawks, D. C. 2002. Jewel scarabs. University of Nebraska State Museum. Museum Notes, 112.

Matsuda, R. 1976. Morphology and evolution of the insect abdomen; with special referente to developmental patterns and their bearing upon systematics. Pergamon Press, Oxford.

Micó, E. 2001. Los escarabeidos antófilos de la península Ibérica (Col. Scarabaeoidea: Hopliinae, Rutelidae, Cetoniidae): taxonomía, filogenia y biología. Tesis Doctoral inédita. Departamento de Ciencias Ambientales y Recursos Naturales, Universidad de Alicante, España. 519 pp.

Monzón-Sierra, J. 2006. El género Chrysina Kirby (Coleoptera: Scarabaeidae) en Guatemala. Pp. 393401. In: Cano, B. E. (ed.) Biodiversidad de Guatemala, volumen I. Universidad del Valle de Guatemala, Guatemala.

Morón, M. A. 1976. Descripción de las larvas de tres especies mexicanas de pelidnotinos (Coleoptera, Melolonthidae, Rutelinae) y algunas observaciones sobre su biología. Anales Instituto de Biología UNAM, serie Zoología, 74(1): 7-18.

Morón, M. A. 1985. Observaciones sobre la biología de dos especies de rutelinos saproxilófagos en la Sierra de Hidalgo, México. (Col. Melolonthidae, Rutelinae). Folia Entomológica Mexicana, 64: 4153.

Morón, M. A. 1990. Rutelini (I): Plusiotis, Chrysina, Chrysophora, Pelidnotopsis, Ectinoplectron. The Beetles of the World No. 10. Sciences Nat. Compiegne, France.

Morón, M. A. 1991. Estudio biogeográfico-ecológico preliminar del género Plusiotis Burmeister (Coleoptera: Melolonthidae: Rutelinae). Giornale italiano di Entomologia, 5: 309-323.

Morón, M. A. 1999. Belleza, diversidad y rareza de escarabajos mexicanos. Biodiversitas (CONABIO), número 26: 1-6.

Morón, M. A. y C. Deloya. 1990. Los Coleoptera Lamellicornia de la Reserva de la Biósfera La Michilía, Durango, México. Folia Entomológica Mexicana, 81: 209-283.

Nogueira, G., M. A. Morón, H. E. Fierros-López \& J. L. Navarrete-Heredia. 2004. The immature stages of Neoscelis dohrni (Westwood) (Coleoptera: Scarabaeidae: Cetoniinae: Goliathini) with notes on adult behavior. The Coleopterists Bulletin, 58(2): 171-183.

Ritcher, P. O. 1966. White grubs and their allies. Oregon State University Press, Corvallis.

Sharp, D. \& F. Muir. 1912. The comparative anatomy of the male genital tube in Coleoptera. Transactions of the Royal Entomological Society of London, 60: 477-642.

Snodgrass, R. E. 1935. Principles of insect morphology. McGraw-Hill Book Company, New York.

Solís, A. 2009. Los escarabajos dorados (Chrysina) de Costa Rica. http://www.inbio.ac.cr/ papers/Plusiotis/Plusiotis.html\#lista. Accesado el 15 de octubre de 2009.

Young, F. N. 1957. Notes on the habits of Plusiotis gloriosa Leconte (Scarabaeidae). The Coleopterists Bulletin, 11: 67-70. 